\title{
A MASSIVE BIPOLAR OUTFLOW AND A DUSTY TORUS WITH LARGE GRAINS IN THE PREPLANETARY NEBULA IRAS 22036+5306
}

\author{
R. SAhai, ${ }^{1}$ K. Young, ${ }^{2}$ N. A. Patel, ${ }^{2}$ C. SÁnchez Contreras, ${ }^{3}$ and M. Morris ${ }^{4}$ \\ Received 2006 June 19; accepted 2006 August 10
}

\begin{abstract}
We report high angular resolution $\left(\sim 1^{\prime \prime}\right)$ CO $J=3-2$ interferometric mapping using the Submillimeter Array (SMA) of IRAS $22036+5306$ (I22036), a bipolar preplanetary nebula (PPN) with knotty jets discovered in our HST snapshot survey of young PPNs. In addition, we have obtained supporting lower resolution $\left(\sim 10^{\prime \prime}\right) \mathrm{CO}$ and ${ }^{13} \mathrm{CO} J=$ 1-0 observations with the Owens Valley Radio Observatory (OVRO) interferometer, as well as optical long-slit echelle spectra at the Palomar Observatory. The CO $J=3-2$ observations show the presence of a very fast $\left(\sim 220 \mathrm{~km} \mathrm{~s}^{-1}\right)$, highly collimated, massive $\left(0.03 M_{\odot}\right.$ ) bipolar outflow with a very large scalar momentum (about $\left.10^{39} \mathrm{~g} \mathrm{~cm} \mathrm{~s}^{-1}\right)$, and the characteristic spatiokinematic structure of bow shocks at the tips of this outflow. The $\mathrm{H} \alpha$ line shows an absorption feature blueshifted from the systemic velocity by $\sim 100 \mathrm{~km} \mathrm{~s}^{-1}$, which most likely arises in neutral interface material between the fast outflow and the dense walls of the bipolar lobes at low latitudes. The fast outflow in I22036, as in most PPNs, cannot be driven by radiation pressure. We find an unresolved source of submillimeter (and millimeterwave) continuum emission in I22036, implying a very substantial mass $\left(0.02-0.04 M_{\odot}\right.$ ) of large (radius $\gtrsim 1 \mathrm{~mm}$ ), cold $\left(\lesssim 50 \mathrm{~K}\right.$ ) dust grains associated with I22036's toroidal waist. We also find that the ${ }^{13} \mathrm{C} /{ }^{12} \mathrm{C}$ ratio in $\mathrm{I} 22036$ is very high $(0.16)$, close to the maximum value achieved in equilibrium $\mathrm{CNO}$ nucleosynthesis $(0.33)$. The combination of the high circumstellar mass (i.e., in the extended dust shell and the torus) and the high ${ }^{13} \mathrm{C} /{ }^{12} \mathrm{C}$ ratio in $\mathrm{I} 22036$ provides strong support for this object having evolved from a massive $\left(\gtrsim 4 M_{\odot}\right)$ progenitor in which hot-bottom-burning has occurred.
\end{abstract}

Subject headings: circumstellar matter — planetary nebulae: individual (IRAS 22036+5306) reflection nebulae - stars: AGB and post-AGB — stars: mass loss - stars: winds, outflows

\section{INTRODUCTION}

Preplanetary nebulae (PPNs), objects in transition between the asymptotic giant branch (AGB) and planetary nebula (PN) evolutionary phases, hold the key to one of the most vexing and long-standing problems in our understanding of these very late stages of evolution for low- and intermediate-mass stars. Current observational evidence shows that during the PPN phase, the dense, isotropic mass loss, which generally marks the late AGB phase, changes to high-velocity bipolar or multipolar mass loss. The emergence of fast collimated outflows or jets has been hypothesized as the primary mechanism for this dramatic change in the geometry and dynamics of the mass loss (Sahai \& Trauger 1998). However, the physical mechanism for producing the fast outflows remains unknown. Submillimeter- and millimeter-wave interferometric observations offer one of the best probes of the dynamics and energetics of the shock acceleration process, which transfers a substantial amount of directed momentum to large parts of the dense AGB wind.

IRAS 22036+5306 (I22036) is a bipolar PPN with knotty jets discovered in our Hubble Space Telescope (HST) snapshot survey of young PPNs, with an estimated distance and luminosity of $2 \mathrm{kpc}$ and $2300 L_{\odot}$, respectively (Sahai et al. 2003; hereafter S03). Our HST imaging suggested that I22036 is a key object for

\footnotetext{
1 Jet Propulsion Laboratory, California Institute of Technology, MS 183-900, Pasadena, CA 91109.

${ }^{2}$ Harvard-Smithsonian Center for Astrophysics, 60 Garden Street, Cambridge, MA 02138.

3 California Institute of Technology, MS 105-24, Pasadena, CA 91125; current address: Departamento de Astrofisica Molecular e Infrarroja, Instituto de Estructura de la Materia, CSIC, Serrano 121, 28006 Madrid, Spain.

4 Division of Astronomy, Department of Physics and Astrophysics, University of California, Los Angeles, CA 90095.
}

understanding how jets can sculpt bipolar lobes in a progenitor AGB star wind, since its jets and their working surfaces appear to be simultaneously observed. In this paper (the second one by Sahai and collaborators on this important PPN) we report CO $J=3-2$ observations of the PPN with the Submillimeter Array (SMA) that resolve a massive high-velocity bipolar outflow aligned with the optical nebulosity and clearly pinpoint the location and nature of the bow shocks at the tips of this outflow. We also report supporting lower resolution $\left(\sim 10^{\prime \prime}\right) \mathrm{CO}$ and ${ }^{13} \mathrm{CO}$ $J=1-0$ observations with the Owens Valley Radio Observatory (OVRO) interferometer, as well as optical long-slit echelle spectra at the Palomar Observatory.

The rest of the paper is organized as follows: in $\S 2$ we describe our observational techniques and data reduction; in $\S 3$ we describe our observational results; in $\S 4$ we discuss the kinematics, age, and mass of the bipolar outflow; in $\S 5$ we derive the total molecular mass and compare it to the mass of dust in the circumstellar environment; in $\S 6$ we derive and discuss the implications of the ${ }^{13} \mathrm{C} /{ }^{12} \mathrm{C}$ isotope ratio in I22036; in $\S 7$ we discuss the formation of the nebula; and in $\S 8$ we summarize our main conclusions.

\section{OBSERVATIONS}

The CO $J=3-2$ observations were obtained with the Submillimeter Array (SMA), using two tracks, on 2004 July 20 (UT) and 2004 August 29 (UT), with the array in the compact and extended configurations, respectively. The J2000.0 coordinates used for I22036 were R.A. $=22^{\mathrm{h}} 05^{\mathrm{m}} 30^{\mathrm{s}} \mathrm{s} 2$, decl. $=+53^{\circ} 21^{\prime} 33^{\prime \prime} .0$. The receivers were tuned to center the $345.796 \mathrm{GHz}$ rest frequency of the $\mathrm{CO}(3-2)$ line in the upper sideband (USB), after correcting for the source's $-45 \mathrm{~km} \mathrm{~s}^{-1}$ local standard of rest (LSR) velocity. On the night of 2004 July 20, the weather was excellent, 
with $0.7 \mathrm{~mm}$ of precipitable water vapor. The antennas' double sideband (DSB) system temperatures ranged from $\sim 200 \mathrm{~K}$ near the zenith to $\sim 350 \mathrm{~K}$ at an elevation of $30^{\circ}$. Unfortunately, one of the eight antennas had extremely high phase noise on that night, and its data were unusable. On the night of 2004 August 29, all eight antennas were working well, but the precipitable water vapor varied between 1.0 and $1.6 \mathrm{~mm}$, causing the system temperatures to range from $\sim 250$ to $\sim 500 \mathrm{~K}$. For both tracks, the correlator was configured to have the highest available uniform resolution, $406.25 \mathrm{kHz}$ channel $\left(0.35 \mathrm{~km} \mathrm{~s}^{-1}\right)$, over a total bandwidth of $2 \mathrm{GHz}$. Observations of Saturn and Uranus were obtained for bandpass calibration, and Titan and Uranus were used for flux calibration. The quasars BL Lac and 2013+370 were observed every $\frac{1}{2} \mathrm{hr}$ for use as complex gain calibrators. A total of $12 \mathrm{hr}$ of on-source data was taken, split nearly evenly between the two tracks.

The data were calibrated using the MIR package, and mapping was done with AIPS. Visibility weights were derived from receiver $T_{\text {sys }}$ measurements. After calibration, the data were exported to AIPS for mapping. Two spectral lines, CO $J=3-2$ and an unexpected second line, were detected in the upper sideband (USB), but no lines were seen in the lower sideband (LSB). A continuum level was fitted to the regions of the USB data that were free of line emission, and was subtracted from the visibility data. Two data cubes were then generated. When producing the first cube, we included data from both tracks with the aim of generating maps of the emission integrated over large velocity intervals with the smallest synthesized beam. For the second, we discarded the more noisy data from the extended track, with the aim of generating high-quality emission maps with much narrower velocity bins. The final data cubes had 4872 channels after the removal of overlapping portions of the spectra lying in the roll-off regions of the hybrid correlator's analog filters. In the first (second) data cube, the clean beam had a FWHM $=1$ ". $12 \times$ $1^{\prime \prime} 00\left(1^{\prime \prime} 45 \times 1^{\prime \prime} .29\right)$ at a position angle (P.A.) of $88^{\circ} .6\left(-84^{\circ} .7\right)$. A continuum map was made using the LSB data and uniform weighting of $T_{\text {sys }}$-weighted visibilities. The central $250 \mathrm{MHz}$ of the LSB IF coverage was rejected, in order to guard against contamination from the strong CO emission in the USB. A $180 \mathrm{k} \lambda$ taper was applied to the visibility data when maps were produced. Uniform weighting was used.

Interferometric observations of the $\mathrm{CO}$ and ${ }^{13} \mathrm{CO} J=1-0$ line emission were obtained at OVRO on 2002 September 21 as part of a snapshot survey of PPNs. General details on the observational setup are given in Sánchez Contreras \& Sahai (2004); data reduction and calibration steps are similar to those described in Sahai et al. (2005b). The clean beam had a FWHM of 9". $\times 5^{\prime \prime} .9$ $\left(\right.$ P.A. $\left.=87^{\circ}\right)$ for $\mathrm{CO}$ and $10^{\prime \prime} .2 \times 66^{\prime \prime} .7$ (P.A. $\left.=82^{\circ}\right)$ for ${ }^{13} \mathrm{CO}$. Scale conversion factors were 1.6 and $1.5 \mathrm{~K}\left(\mathrm{Jy} \mathrm{beam}^{-1}\right)^{-1}$ for $\mathrm{CO}$ and ${ }^{13} \mathrm{CO}$.

Since I22036 displays a prominent $\mathrm{H} \alpha$ emission line, and the presence of a fast neutral outflow can produce a self-absorption signature in this line (e.g., Sánchez Contreras \& Sahai 2001), we also obtained a high-resolution $(\Delta \lambda=0.4 \AA)$ spectrum of I22036 in order to resolve the velocity structure of the line, using the Palomar 60 inch $(1.52 \mathrm{~m})$ spectrograph in echelle mode. In this mode, the spectrograph provided a total wavelength coverage of $\sim 3600-9000 \AA$, with a resolution of about 19,000 . The echelle grating had 52.65 lines $\mathrm{mm}^{-1}$ and a blaze angle of $63^{\circ} 26^{\prime}$. Cross-dispersion was provided by two fused quartz prisms with a $60^{\circ}$ apex angle. The slit was 7".4 long and had a width of 1".4. The spectra were recorded on a thinned, lumogen-coated TI $800 \times$ 800 chip. A thorium-argon lamp was used for wavelength calibration, and the standard star HR 7596 was used for flux calibra- tion. The data were obtained during the nights of 2002 August 4 and 5, and were reduced and calibrated using standard IRAF techniques.

\section{RESULTS}

\subsection{Spectral Profiles}

The (spatially) averaged CO $J=3-2$ line profile shows a strong central core and weaker extended wings (Fig. 1a). The wings cover a total velocity extent (FWZI) of $\sim 450 \mathrm{~km} \mathrm{~s}^{-1}$ and are most likely associated with a fast post-AGB outflow. The large width $\left(\mathrm{FWHM} \approx 70 \mathrm{~km} \mathrm{~s}^{-1}\right)$ of the central core of the CO $J=3-2$ emission indicates that much of the core emission is also associated with the fast outflow.

As with the $J=3-2$ line profile, the $\mathrm{CO}$ and ${ }^{13} \mathrm{CO} J=1-0$ line profiles obtained also show wide wings $\left(\gtrsim 100 \mathrm{~km} \mathrm{~s}^{-1}\right)$ but do not cover as wide a velocity range (Fig. $1 b$ ). The observed extent of the $\operatorname{CO} J=1-0$ line wings is limited by the spectrometer bandwidth $\left(\approx 200 \mathrm{~km} \mathrm{~s}^{-1}\right)$, whereas that of the ${ }^{13} \mathrm{CO}$ line wings is sensitivity limited. The $J=1-0$ line profile has a triangular shape with a total velocity extent (FWZI) of greater than $100 \mathrm{~km} \mathrm{~s}^{-1}$, with no obvious central core. It is likely that this $J=1-0$ emission is associated with the material seen in the central core of the $J=3-2$ line. The ${ }^{13} \mathrm{CO} J=1-0$ line shows a narrow central core, with a width of about $20 \mathrm{~km} \mathrm{~s}^{-1}$, and weaker wings extending over a similar velocity range as the $\mathrm{CO} J=1-0$ line. Since the typical expansion velocities of AGB circumstellar envelopes (CSEs) are 10-15 $\mathrm{km} \mathrm{s}^{-1}$, the narrow central core seen in the ${ }^{13} \mathrm{CO} J=1-0$ line may be associated with the remnant progenitor AGB envelope in I22036, or slowly expanding gas in the dense waist.

In addition to the $\mathrm{CO} J=3-2 \mathrm{CO}$ line, we detected a line at a rest frequency of $346.53 \mathrm{GHz}$, most likely due to the SO $N=8-7, J=9-8$ transition with a frequency of 346.5284810 $\mathrm{GHz}$ (JPL catalog). The observed SO line is much narrower than the CO line, with a full width of $21 \mathrm{~km} \mathrm{~s}^{-1}$, and an integrated flux of $20 \mathrm{Jy} \mathrm{km} \mathrm{s}^{-1}$ (Fig. 1c). The SO emission appears to be slightly elongated and extended over $\sim 2^{\prime \prime}$, with a P.A. of about $-30^{\circ}$ (Fig. 2, top left). Within the relatively low signal-to-noise ratio, the red and blue wings of the line are colocated, indicating that the SO emission does not arise in a bipolar outflow. Given its similar velocity width compared to the narrow core of the ${ }^{13} \mathrm{CO}$ 1-0 line, the SO emission is most likely associated with the same material as the former.

The $\mathrm{H} \alpha$ line shows a profile with a central peak, very wide wings, and an absorption dip in the blue wing of the line, centered at $V_{\mathrm{LSR}}$ of about $-145 \mathrm{~km} \mathrm{~s}^{-1}$ (Fig. $1 d$ ). This peculiar $\mathrm{H} \alpha$ line shape is seen in many PPNs and young, compact PNs (van de Steene et al. 2000; Sánchez Contreras \& Sahai 2001; Sahai \& Sánchez Contreras 2004; Sahai et al. 2005b). The blue and red wings can be seen extending to velocities of roughly -400 and $500 \mathrm{~km} \mathrm{~s}^{-1}$, respectively. The $\mathrm{H} \alpha$ line profile (e.g., IRAS $19024+0044$, Hen 3-1475) is most simply interpreted in terms of a model in which the (spectrally) broad $\mathrm{H} \alpha$ emission arises in a compact, spatially unresolved central source, ${ }^{5}$ and this emission and stellar continuum is scattered by dust in the extended, expanding nebula. The blueshifted absorption arises due to a largely atomic outflow within the foreground lobe, which absorbs the scattered photons. The scattering is expected to produce an overall redshift of the $\mathrm{H} \alpha$ line peak relative to the systemic radial

\footnotetext{
5 The nature of this source is unknown, although possible mechanisms include emission from a very high velocity outflow, Raman scattering, and/or Keplerian rotation in a dense disk.
} 

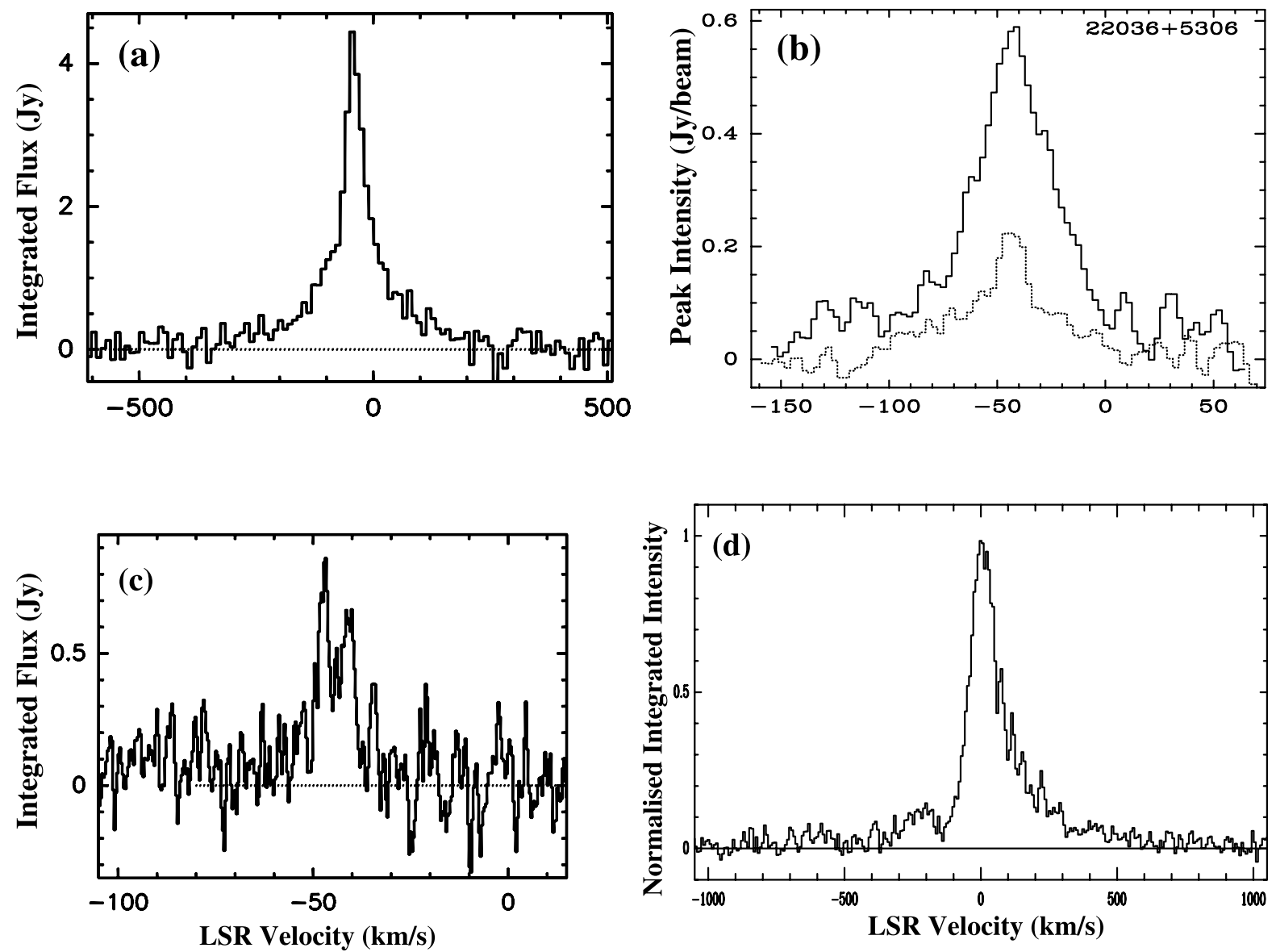

FIG. 1.- Spectra of the $(a) \mathrm{CO} J=3-2$ line integrated over the entire emission region, $(b) \mathrm{CO}$ and ${ }^{13} \mathrm{CO} 1-0$ peak intensity in the unresolved emission source for each line, $(c)$ the SO 9(8)-8(7) line integrated within a $2^{\prime \prime} \times 2^{\prime \prime}$ area centered on the SO peak (5 channel Hanning smoothing has been applied), and $(d)$ the $\mathrm{H} \alpha$ line flux $\left(F_{\lambda}\right.$, normalized to a peak value of unity), in IRAS $22036+5306$

velocity, as observed; the peak of the $\mathrm{H} \alpha$ line is located at $V_{\mathrm{LSR}} \approx 0 \mathrm{~km} \mathrm{~s}^{-1}$, redshifted from the systemic velocity, $V_{\mathrm{LSR}}=$ $-45 \mathrm{~km} \mathrm{~s}^{-1}$. Such a model has been successfully used to quantitatively explain the spatiokinematic distribution of similar $\mathrm{H} \alpha$ emission profiles observed with the HST Space Telescope Imaging Spectrograph (STIS) in the PPN Hen 3-1475 (Sánchez Contreras \& Sahai 2001).

\subsection{Velocity Channel Maps}

We show the spatial distribution of the $\mathrm{CO} J=3-2$ emission as a function of velocity in Figure 2. The centroid of the emission is seen to shift steadily from west to east in the panels as the average velocity decreases from 217 to $-217 \mathrm{~km} \mathrm{~s}^{-1}$, indicating a strong velocity gradient along the major axis of the nebula. A plot of the central $\pm 15 \mathrm{~km} \mathrm{~s}^{-1}$ range with narrower velocity bins of width $2.47 \mathrm{~km} \mathrm{~s}^{-1}$ (Fig. 3) also shows roughly an east-west velocity gradient. We cannot separate a kinematically distinct component in the $J=3-2$ emission in the velocity range covered by the central core of the ${ }^{13} \mathrm{CO} J=1-0$ line. The overall velocity gradient can be seen more easily in a plot of the blue and red wing emission of the $\mathrm{CO} J=3-2$ line, overlaid on the optical image of I22036; there is a large separation between the red- and blueshifted emission, implying the presence of a bipolar outflow (Fig. 4) along the long axis of I22036. In order to register the HST and SMA images, we first rotated the latter to the orientation of the former. For the final fine translational registration, we assumed that peak "C" $(\mathrm{S} 03)$ coincides with the peak of the integrated $\mathrm{CO} J=3-2$ intensity (shown in Fig. 5), since the absolute astrometry of the $H S T$ images, relative to that of the SMA images, cannot be determined to a sufficiently high accuracy (i.e., better than a few $\times 0$ "' 1 ). The outflow is tilted such that the west lobe (east lobe) is on the near (far) side; this tilt is consistent with that inferred from the difference in brightness between the two optical lobes and the morphology of the waist (S03). We have not shown the corresponding integrated intensity or velocity channel maps for the OVRO observations, because these do not resolve the $\mathrm{CO} J=1-0$ emission due to the large beamwidth.

The map of the emission integrated over all velocities in Figure 5 shows that the emission lies in an elongated region, oriented along the major axis of the optical nebula. A central, unresolved continuum source (at $\lambda=0.88 \mathrm{~mm}$ ) with a flux of $0.29 \pm 0.04 \mathrm{Jy}$ is also seen; the error is dominated by calibration uncertainties. The peak of the continuum source appears to be offset from the line intensity peak by $\sim 0{ }^{\prime \prime} 23$. An unresolved continuum source is also detected in the OVRO data, with a flux of $8.4 \pm 1.7 \mathrm{mJy}$.

\subsection{Position-Velocity Structure}

A position-velocity (PV) plot along the bipolar axis (Fig. 6) shows abrupt increases in radial velocity at offsets of about $\pm 1^{\prime \prime}$ from the center. Such structure is typical of what is expected when a high-velocity bipolar collimated outflow or jet (hereafter simply referred to as the jet) interacts with dense ambient material, producing bow shocks at the head of the jet (see, e.g., models and right panel of Fig. 2 in Lee \& Sahai 2003; hereafter LS03). The bow shocks implied by the $\mathrm{CO}$ velocity structure 


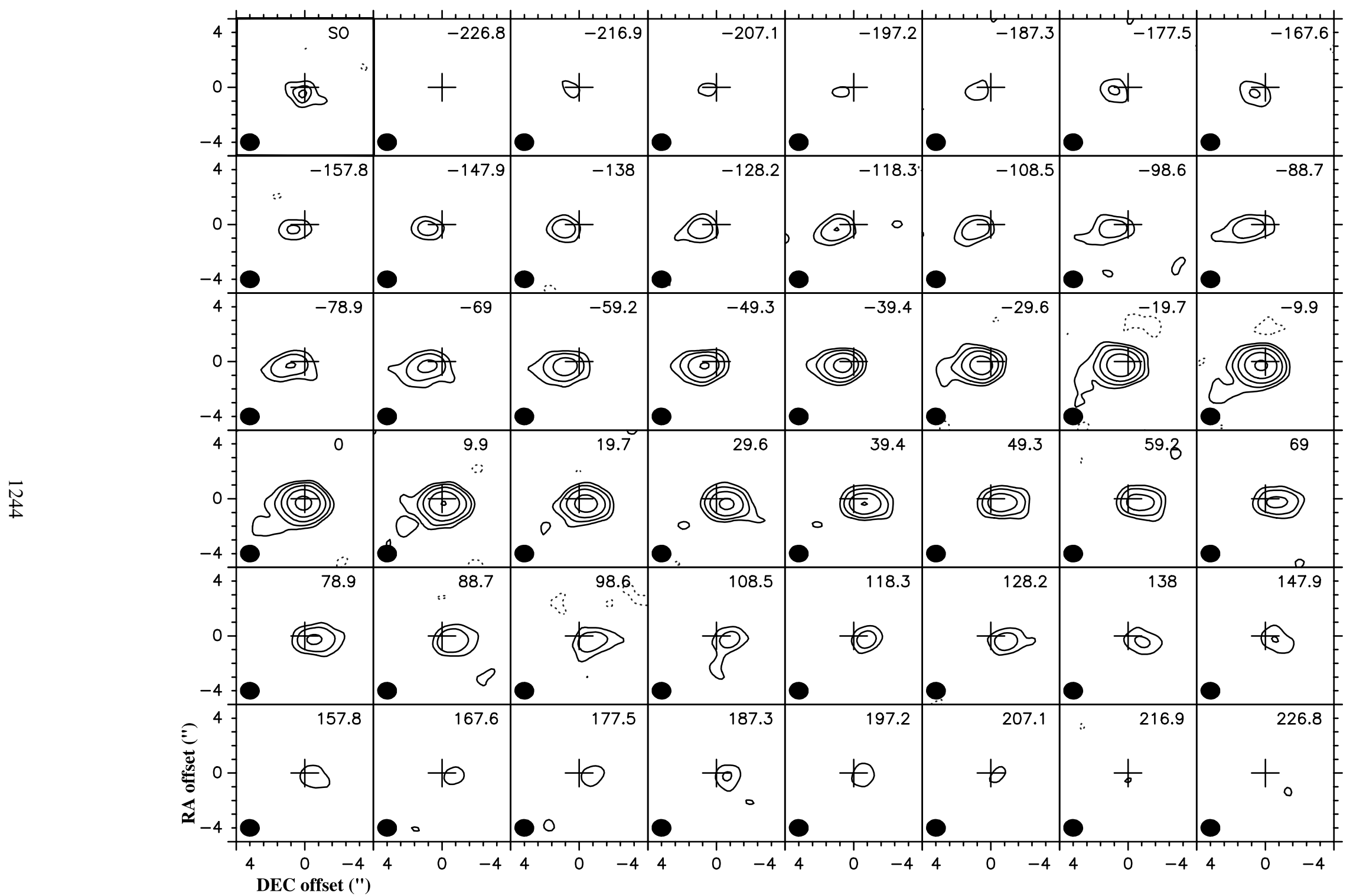

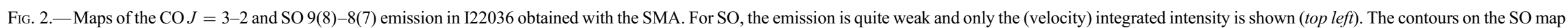

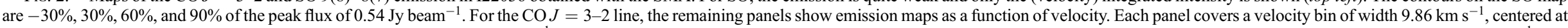

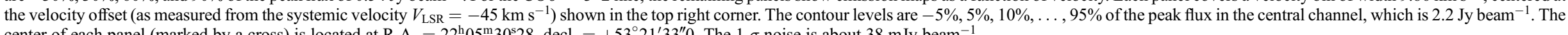
center of each panel (marked by a cross) is located at R.A. $=22^{\mathrm{h}} 05^{\mathrm{m}} 30^{\mathrm{s}} .28$, decl. $=+53^{\circ} 21^{\prime} 33^{\prime \prime} .0$. The $1 \sigma$ noise is about $38 \mathrm{mJy}$ beam ${ }^{-1}$. 


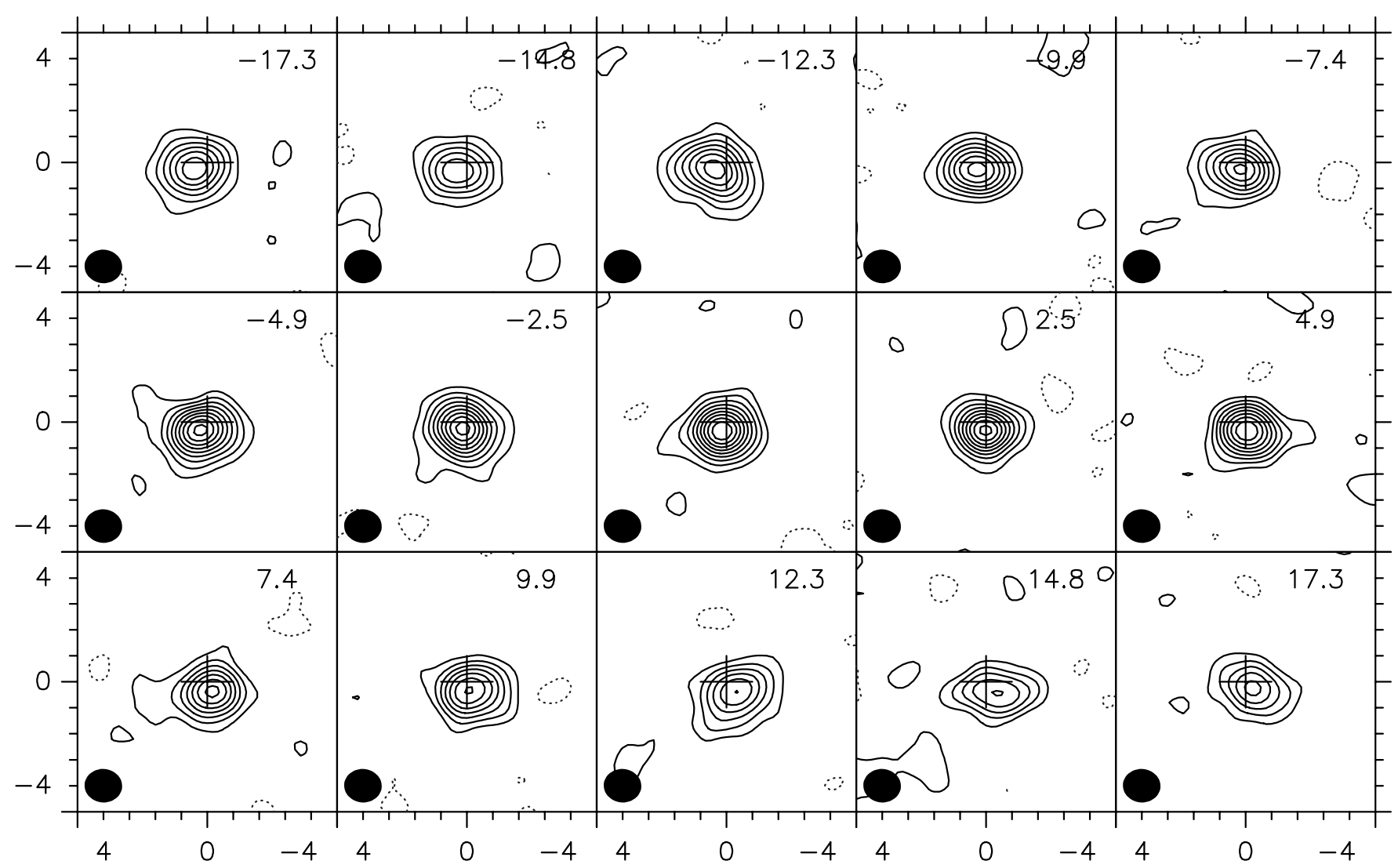

FIG. 3.- Same as in Fig. 2, but with the total velocity coverage extending over the central $\pm 15 \mathrm{~km} \mathrm{~s}^{-1}$ range, with each panel showing emission in a velocity bin of width $2.47 \mathrm{~km} \mathrm{~s}^{-1}$. Contour levels are $-10 \%, 10 \%, 20 \%, \ldots, 90 \%$ of the peak flux in the central channel, which is $2.2 \mathrm{Jy}$ beam ${ }^{-1}$.

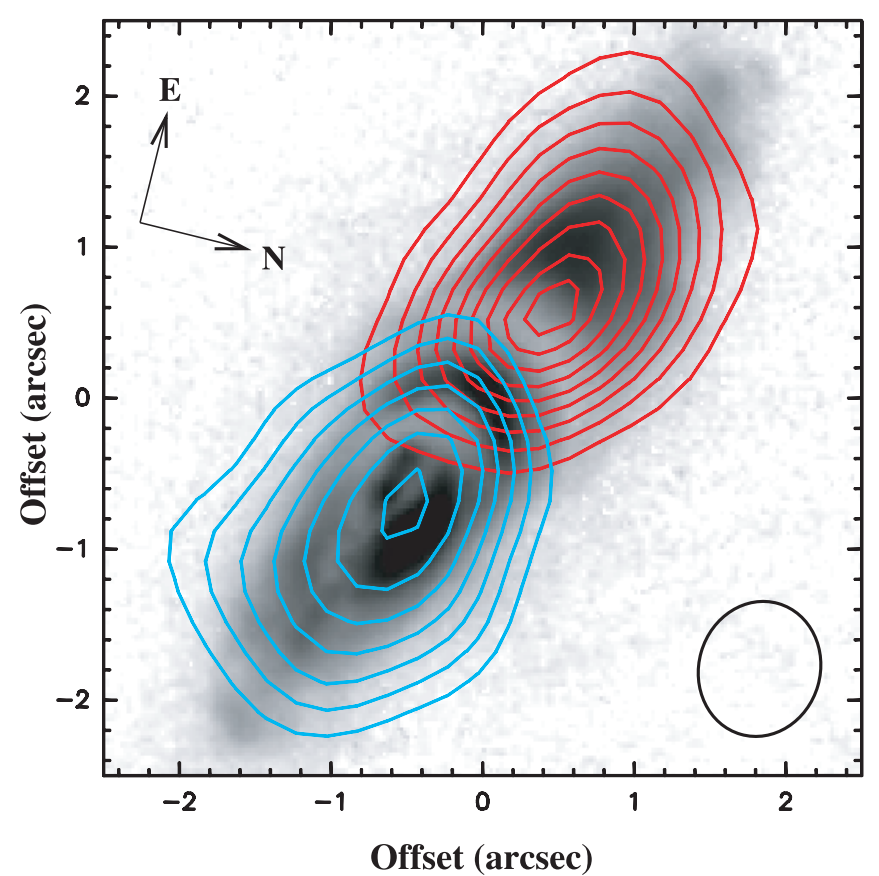

FIG. 4.- Map of the blue and red wing CO $J=3-2$ line emission from IRAS $22036+5306$, overlaid on a $0.6 \mu \mathrm{m} H S T$ image. The velocity ranges used to define the blue and red wings in this plot are offset by -245 to $-61 \mathrm{~km} \mathrm{~s}^{-1}$, and $61 \mathrm{~km} \mathrm{~s}^{-1}$ to $245 \mathrm{~km} \mathrm{~s}^{-1}$ from the systemic velocity of $-45 \mathrm{~km} \mathrm{~s}^{-1}$. The peak surface brightness in the red (blue) lobe is 4.10 (3.37) $\mathrm{Jy} \mathrm{km} \mathrm{s}^{-1}$ beam ${ }^{-1}$. Contours are $0.82,1.23,1.64, \ldots, 3.28 \mathrm{Jy} \mathrm{km} \mathrm{s}^{-1}$ beam $^{-1}$ in the blue lobe, (i.e., with a step of $0.41 \mathrm{Jy} \mathrm{km} \mathrm{s}^{-1}$ beam ${ }^{-1}$ ), and $0.82,1.23,1.64, \ldots, 3.69$, and $3.95 \mathrm{Jy} \mathrm{km} \mathrm{s}^{-1}$ beam $^{-1}$ in the red lobe. The size and orientation of the clean beam $\left(1^{\prime \prime} 12 \times 1\right.$ ". 00, P.A. $=$ $88^{\circ}$ ) are shown in the lower right corner.

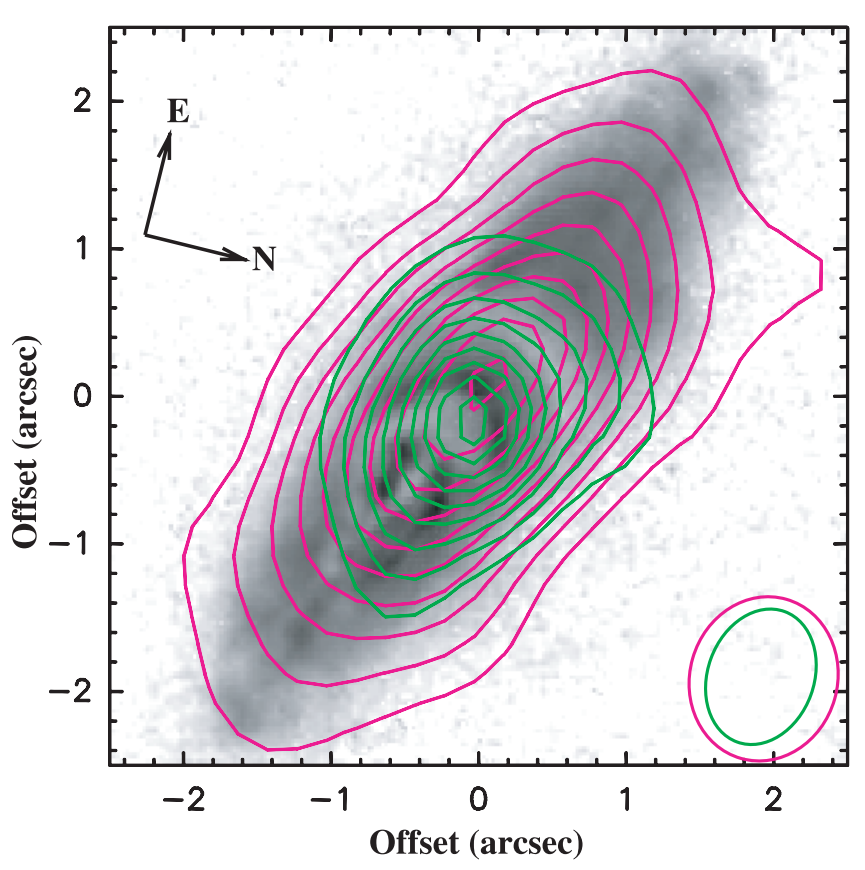

FIG. 5.-Maps of the velocity-integrated CO $J=3-2$ line emission (magenta contours; levels $15 \%-95 \%$ of the peak emission $\left[13.3 \mathrm{Jy} \mathrm{km} \mathrm{s}^{-1}\right.$ beam $\left.^{-1}\right]$ in steps of $10 \%$ ) and $0.88 \mathrm{~mm}$ continuum emission (green contours; levels $15 \%-95 \%$ of the peak emission $\left[0.17 \mathrm{Jy}\right.$ beam $^{-1}$ ] in steps of $10 \%$ ) from IRAS $22036+5306$, obtained with the SMA, overlaid on a $0.6 \mu \mathrm{m} H S T$ image (processed to enhance sharp features, as in S03). The $1 \sigma$ noise in the continuum map is $4.5 \mathrm{mJy} \mathrm{beam}^{-1}$. The sizes and orientations of the clean beams for the line maps $(1$ ". $12 \times 1$ ". 00 , P.A. $=$ $\left.88^{\circ} .6\right)$ and continuum maps $\left(0.94 \times 0^{\prime \prime} .72\right.$, P.A. $\left.=83^{\circ} .9\right)$ are shown in the lower right corner. 


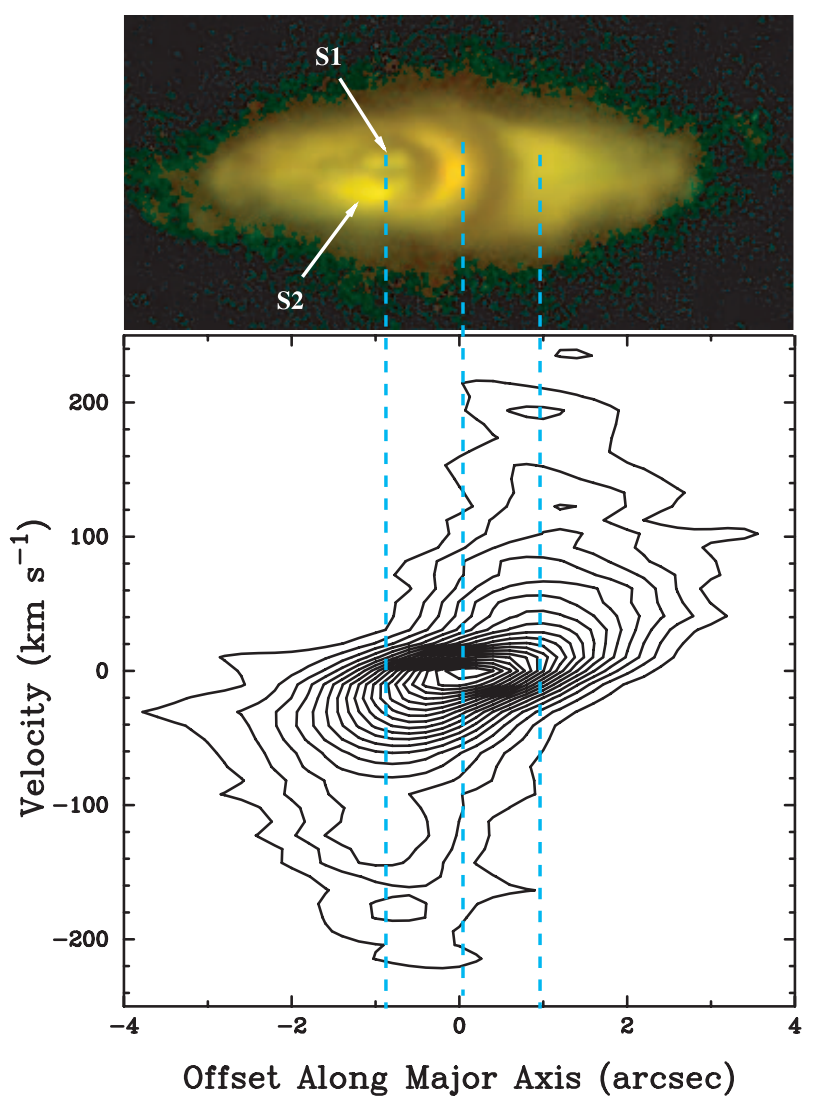

FIG. 6.-PV plot of the $\mathrm{CO} J=3-2$ emission along the major axis of the nebula. Contour levels are $5 \%-95 \%$ of the peak in steps of $5 \%$. A color $H S T$ image of the nebula (with the $0.6 \mu \mathrm{m}$ image in green and the $0.8 \mu \mathrm{m}$ image in red, as in S03) is shown, with its major axis aligned along the spatial axis in the PV plot, for comparison.

appear to be located in the general vicinity of, and may be identified with, the bright regions S1, S2 in the west lobe and their presumed optically obscured counterparts in the east lobe. The PV structure has a high degree of symmetry around the center, indicating that the bipolar outflow and surrounding medium are correspondingly symmetric.

In addition to the high-velocity bow-shock structures, the PV diagram shows a weaker and significantly more spatially extended component, which extends out to the tips of the optical bipolar nebula. This component is composed of relatively low-velocity emission, but the limits of its velocity structure at the high end cannot be separated from the bow-shock emission.

There are two possible explanations for the simultaneous presence of the low- and high-velocity emission structures, with (1) their different spatial extents seen in the $\mathrm{CO} J=3-2 \mathrm{PV}$ plot and (2) correspondence to the morphological features seen in the optical images. One possibility is that the extended lobes in I22036 are geometrically distinct from the outflow that produces the high-velocity emission and the bright optical knots S1 and S2. The latter is probably a collimated outflow whose axis is tilted significantly toward the observer, whereas the extended lobes are more in the plane of the sky (assuming that the three-dimensional [3D] outflow velocity of the material in these lobes is not too different from that in the high-velocity outflow). So in 3D, I22036 may be a quadrupolar nebula, with the near-side lobe representing the $\mathrm{S} 1, \mathrm{~S} 2$ outflow seen in projection in front of the extended lobe. A second possibility is that the extended lobes have a similar inclination as the material in the fast bipolar outflow, and

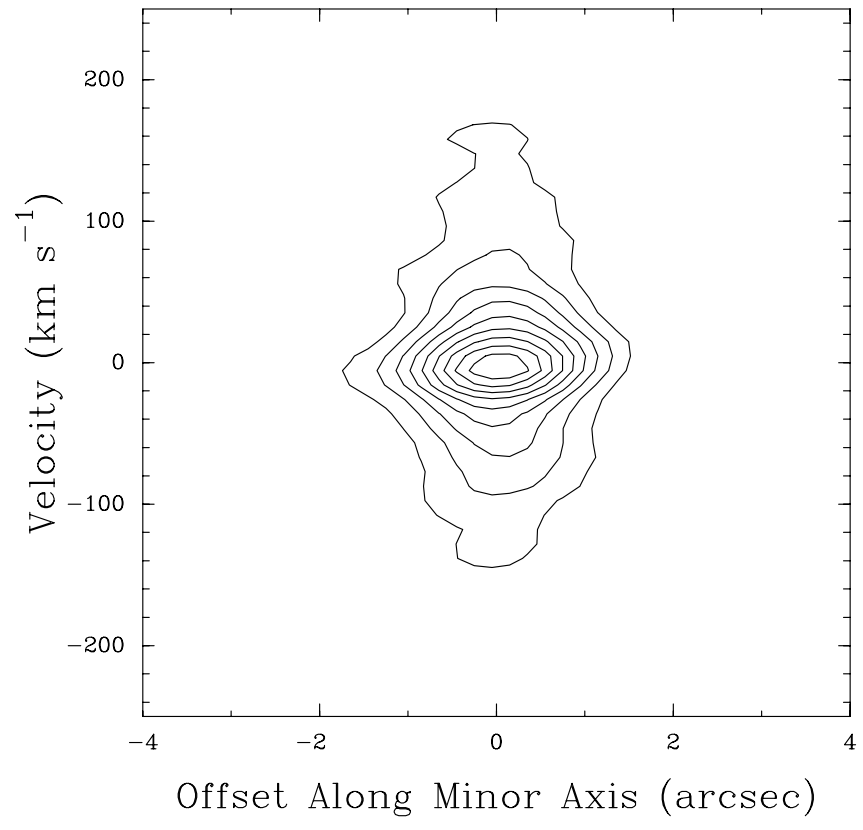

FIG. 7.-PV plot of the $\mathrm{CO} J=3-2$ emission along the minor axis of the nebula. Contour levels are $10 \%, 20 \%, \ldots, 90 \%$ of the peak in steps of $10 \%$.

the emission with low (projected) velocity in the PV plot comes from an outflow that is intrinsically slower than the former.

A PV plot along the minor axis of the I22036 shows no significant gradient (Fig. 7). This indicates the lack of significant rotation or bipolar outflow motions in the region of the dusty nebular waist.

\section{THE POST-AGB BIPOLAR OUTFLOW}

\subsection{Kinematics and Expansion Age}

We have evidence of fast outflows in I22036 from three independent probes. Two of these are the $\mathrm{CO}$ and $\mathrm{H} \alpha$ data in this paper, and a third is the Very Large Array (VLA) mapping of $\mathrm{OH}$ maser emission reported in S03 and Zijlstra et al. (2001). The $\mathrm{OH}$ maser features cover a total velocity spread of about $76 \mathrm{~km} \mathrm{~s}^{-1}$, and are roughly aligned along the nebular axis, with a spatial extent comparable to the distance between the CO-identified bow-shock locations. The PV signature of the $\mathrm{OH}$ is thus similar to that of $\mathrm{CO}$ except at the bow-shock locations, where there is an absence of $\mathrm{OH}$ emission extending to the large outflow velocities seen in $\mathrm{CO}$. Either $\mathrm{OH}$ is destroyed or the appropriate conditions for exciting observable maser emission are not met, in and near the bow-shock region.

Both the $\mathrm{OH}$ and $\mathrm{CO}$ emission presumably come from molecular gas in the dense walls of an elongated lobe, presumably resulting from the interaction of a jet with an ambient circumstellar envelope, as shown by LS03. In contrast to $\mathrm{OH}$ and $\mathrm{CO}$ emission, the $\mathrm{H} \alpha$ absorption probes atomic gas. Since the $\mathrm{H} \alpha$ absorption feature is not spatially resolved in our data, it is difficult to definitively establish its relationship to the fast molecular outflow. In the jet-envelope interaction model, the atomic gas may be located in the interior of the lobes, representing the pristine, unshocked material of the underlying jet, and/or the interface between the latter and the lobe walls. We would thus expect the observed velocity of this atomic material to be generally larger than that of the dense material everywhere in the lobe. But the blueshift of the $\mathrm{H} \alpha$ absorption feature from the systemic velocity implies a projected outflow speed of about $100 \mathrm{~km} \mathrm{~s}^{-1}$, which is smaller than that seen in the $\mathrm{CO}$ at the bow shocks. This 
discrepancy can be explained in the context of the LS03 models, if the $\mathrm{H} \alpha$ absorption feature is produced in interface material (but not "pristine," unshocked jet material) at low latitudes in the outflow lobe, since the velocity of the interface material near the lobe walls is found to decrease with latitude (where the outflow axis represents the polar axis), whereas that of the unshocked jet is constant.

The LS03 modeling shows that, in the momentum-driven case for a jet-envelope interaction, the velocity field of much of the dense material in the resulting elongated lobe is predominantly radial; the age of the outflow is well approximated by dividing the radial distance of the bow shock at the lobe head by the radial velocity of the material there. If we take $220 \mathrm{~km} \mathrm{~s}^{-1}$ and $1^{\prime \prime}$, respectively, as the projected radial velocity and radial distance of the material at the bow shock, based on Figure 6, and if we assume an intermediate inclination angle $\left(i=30^{\circ}\right)$ of the outflow axis to the sky plane, then the fast outflow age is only $25(\tan i / 0.58)$ yr. $^{6}$

\subsection{High-Velocity Outflow: Mass and Scalar Momentum}

We now estimate the mass, $M_{\text {bip }}$, and scalar momentum, $P_{\text {sc }}$, in the high-velocity bipolar outflow from the integrated $J=3-2$ line profile shown in Figure 1 $a$. In this analysis, we have used the $J=3-2$ emission flux over a velocity range extending from 424 $(-505)$ to $15(-15) \mathrm{km} \mathrm{s}^{-1}$ from the systemic velocity for the red (blue) component of the outflow. We have thus excluded the emission in a velocity range $\pm 15 \mathrm{~km} \mathrm{~s}^{-1}$ centered on the systemic velocity, which, as suggested by the ${ }^{13} \mathrm{CO} J=1-0$ and the SO line profiles, might come from a kinematic component distinct from the bipolar outflow. We find values of the velocityintegrated $J=3-2$ emission flux over the blue and red components of the outflow to be 168.3 and $192.5 \mathrm{Jy} \mathrm{km} \mathrm{s}^{-1}$, respectively.

\subsubsection{Excitation Temperatures and Optical Depths}

We need to determine the excitation temperature and evaluate any optical depth effects in order to compute the mass of emitting material. The ratio of the integrated fluxes of the $\mathrm{CO} J=1-0$ and $3-2$ lines, $R 13$, over the high-velocity range covered by both the OVRO and SMA observations (i.e., an interval of $215 \mathrm{~km} \mathrm{~s}^{-1}$ centered at the systemic velocity, but excluding the central $\pm 15 \mathrm{~km} \mathrm{~s}^{-1}$ ) is 0.081 , lower than the value in the central $\pm 15 \mathrm{~km} \mathrm{~s}^{-1}$, which is 0.12 . For optically thin emission in both $J=1-0$ and $2-1$, $R 13=0.081$ implies an excitation temperature $T_{\mathrm{ex}}=14.7 \mathrm{~K}$ and an emitting mass of $0.022 M_{\odot}$. However, if either or both lines are not optically thin, then the inferred temperature and mass will be different.

In order to accurately estimate optical depths as a function of velocity, a detailed spatiokinematic model is needed; however, we do not attempt that here since the emission is not sufficiently well resolved in our data, and so the detailed geometrical structure of the emission is poorly known. Nevertheless, rough estimates of the optical depths can be made by considering the sizes, total fluxes, and total velocity dispersion of the low- and highvelocity components. We first deconvolve the $J=3-2$ line profile into a central Gaussian with a width (FWHM) of $30 \mathrm{~km} \mathrm{~s}^{-1}$, which represents the low-velocity component, and two pairs of high-velocity Gaussian components (HV1r, HV1b and HV2r, HV2b) whose sum represents most of the emission from the

\footnotetext{
${ }^{6}$ The much longer estimate of the age $\left(250 \mathrm{yr}\right.$ for an inclination of $\left.30^{\circ}\right)$ given by $\mathrm{S} 03$ was due to (1) the use of a factor of 5 smaller outflow speed based on the $\mathrm{OH}$ data, and (2) its application to knot $K_{1}$, which is located at a radius about twice as large as that of the bow shocks.
}

high-velocity component. Assuming that the centers of the near (far) pair, HV1r and HV1b (HV2r and HV2b), are symmetrically offset from the systemic velocity by $\pm \mathrm{V} 1( \pm \mathrm{V} 2)$, and that their width is W1 (W2), we find the values of V1, V2, W1, and W2, as well as the peak intensities of the Gaussians that provide the best fit to the $J=3-2$ line. We find $\mathrm{W} 1 \sim 92 \mathrm{~km} \mathrm{~s}^{-1}$ and W2 $\sim 220 \mathrm{~km} \mathrm{~s}^{-1}$. The total flux in the low-velocity component is $100 \mathrm{Jy} \mathrm{km} \mathrm{s}^{-1}$, whereas HV1 and HV2 have about 191 and $170 \mathrm{Jy} \mathrm{km} \mathrm{s}^{-1}$ each.

The angular extent of the high-velocity component is estimated to be about 1".2, i.e., $3.6 \times 10^{16} \mathrm{~cm}$ from the $J=3-2$ blue and red wing emission maps (Fig. 4). In this estimation, we have used geometric means of the major and minor axis of both the emission regions and the beam to obtain deconvolved sizes. Although the detailed spatiokinematic structure of the emitting regions is not known, if we assume that the large velocity gradient approximation is valid, we can obtain rough estimates of the optical depths. We have simulated a large velocity gradient by assuming a spherically expanding cloud with constant expansion velocity equal to half the FWHM of the model components determined above. Such a cloud has significant velocity gradients along most lines of sight (except those which pass very close to the center). For each of the four high-velocity Gaussian components above, we have computed the optical depths (and thus the masses) and excitation temperatures needed to fit the $R 13$ ratio and produce half the $\mathrm{CO} J=3-2$ flux of each component (since the fractional flux within the FWHM points of the 2-dimensional [2D] Gaussians representing each of the HV1 and HV2 components is 0.5 ). These masses are then scaled up by a factor of 2 in order to obtain the mass corresponding to the total flux in each of the HV components.

We have assumed a fractional $f_{\mathrm{CO}}=\mathrm{CO} / \mathrm{H}_{2}$ abundance of $2 \times 10^{-4}$ (S03). For each of the red and blue parts of the HV1 (HV2) component, we find $T_{\mathrm{ex}}=18$ (15.5) $\mathrm{K}$, line-of-sight $J=3-2$ optical depth at a radius half the radius of the model cloud, $\tau_{32}$ of $1.1(0.35)$, and a mass of $4.4 \times 10^{-3}\left(3.1 \times 10^{-3}\right) M_{\odot}$. Adding together the HV1 and HV2 masses from the red and blue parts, and doubling the resulting value to account for the mass contributing to the flux outside the half-power points of the Gaussian fits to these components, we get a total emitting mass in the high-velocity outflow of $M_{\text {bip }}=0.03 M_{\odot}$. (See Table 1, which summarizes our quantitative results for the various physical components in I22036.)

In order to calculate the scalar momentum, we have used the formulation as described in Bujarrabal et al. (2001), assuming optically thin emission and a single temperature for the highvelocity outflow, which we take to be the emission-weighted average ( $17 \mathrm{~K})$ of the HV1 and HV2 components above. We find $P_{\mathrm{sc}}=1.1 \times 10^{39} \mathrm{~g} \mathrm{~cm} \mathrm{~s}^{-1}$ for an intermediate inclination angle $\left(i=30^{\circ}\right)$ of the nebular axis to the sky plane. This outflow cannot be driven by radiation pressure; the dynamical timescale of the lobes, for $i=30^{\circ}$, is only $\sim 25 \mathrm{yr}$, much smaller than the time required by radiation pressure to accelerate the observed bipolar outflow to its current speed, $t_{\mathrm{rad}} \gtrsim P_{\mathrm{sc}} /(L / c) \sim 10^{5} \mathrm{yr}$, given I22036's luminosity of $2300 L_{\odot}$. The value of $t_{\text {rad }}$ is a lower limit because only a fraction of the total luminosity, namely, that directed into the solid angle representing the opening angle of the bipolar outflow, may be utilized for driving the latter.

\section{MOLECULAR MASS VERSUS DUST SHELL MASS}

The size of the low-velocity component is estimated to be about 1."3 $3\left(4 \times 10^{16}\right)$, from the $J=3-2$ emission map in Figure 3 . The flux and angular size of this component are comparable to that in each of the red and blue components in the HV1 and HV2 
TABLE 1

IRAS 22036+5306 Model Components

\begin{tabular}{|c|c|c|}
\hline Parameter & Value & Comments \\
\hline \multicolumn{3}{|c|}{ Cool, Dense Shell } \\
\hline$R_{\text {in }}$ & $1.4 \times 10^{17} \mathrm{~cm}$ & S03 \\
\hline 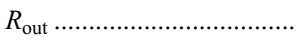 & $5.3 \times 10^{17} \mathrm{~cm}$ & \\
\hline 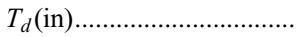 & $67 \mathrm{~K}$ & \\
\hline 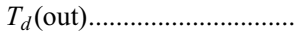 & $35 \mathrm{~K}$ & \\
\hline 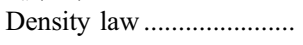 & $r^{-2}$ & \\
\hline 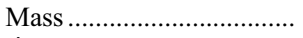 & $4.7 M_{\odot}$ & \\
\hline$\dot{M}$ & $5.5 \times 10^{-4} M_{\odot} \mathrm{yr}^{-1}$ & \\
\hline Grain size .......................... & MRN & \\
\hline \multicolumn{3}{|c|}{ Warm Shell } \\
\hline$R_{\mathrm{in}} \ldots \ldots \ldots \ldots \ldots$ & $1.3 \times 10^{16} \mathrm{~cm}$ & S03 \\
\hline 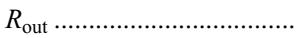 & $1.9 \times 10^{16} \mathrm{~cm}$ & \\
\hline 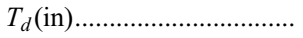 & $145 \mathrm{~K}$ & \\
\hline 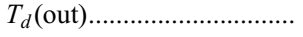 & $82 \mathrm{~K}$ & \\
\hline 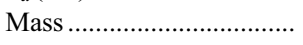 & $5.5 \times 10^{-3} M_{\odot}$ & \\
\hline Grain size ........................... & MRN & \\
\hline
\end{tabular}

Inner Torus/Disk

\begin{tabular}{|c|c|c|}
\hline$R_{\text {in }}$ & $3 \times 10^{14} \mathrm{~cm}$ & S03 \\
\hline 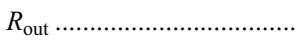 & $4.5 \times 10^{14} \mathrm{~cm}$ & \\
\hline 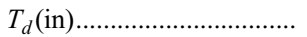 & $1000 \mathrm{~K}$ & \\
\hline 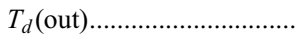 & $365 \mathrm{~K}$ & \\
\hline 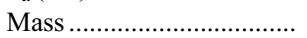 & $6 \times 10^{-4} M_{\odot}$ & \\
\hline 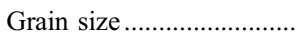 & MRN & \\
\hline
\end{tabular}

Toroid

\begin{tabular}{|c|c|c|}
\hline$R_{\text {in }}$ & $9 \times 10^{15} \mathrm{~cm}$ & This paper \\
\hline 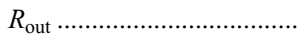 & $1.8 \times 10^{16} \mathrm{~cm}$ & \\
\hline 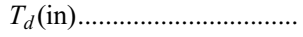 & $50 \mathrm{~K}$ & \\
\hline 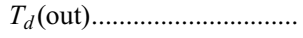 & $35 \mathrm{~K}$ & \\
\hline Mass (dust) ........................ & $0.02-0.04 M_{\odot}$ & \\
\hline Grain size ......................... & $a \gtrsim 1 \mathrm{~mm}$ & \\
\hline
\end{tabular}

Fast Molecular Outflow

\begin{tabular}{|c|c|c|}
\hline 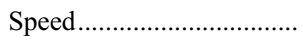 & $\sim 220 \mathrm{~km} \mathrm{~s}^{-1}$ & This paper \\
\hline 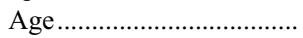 & $25 \mathrm{yr}$ & \\
\hline 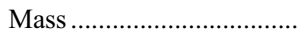 & $0.030 M_{\odot}$ & \\
\hline 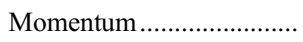 & $9.2 \times 10^{38} \mathrm{~g} \mathrm{~cm} \mathrm{~s}^{-1}$ & \\
\hline 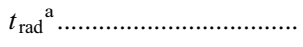 & $\gtrsim 10^{5} \mathrm{yr}$ & \\
\hline
\end{tabular}

Slow Molecular Outflow

\begin{tabular}{|c|c|c|}
\hline 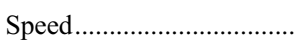 & $\sim 15 \mathrm{~km} \mathrm{~s}^{-1}$ & This paper \\
\hline 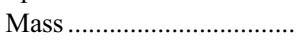 & $0.035 M_{\odot}$ & \\
\hline
\end{tabular}

Fast Atomic Outflow

$\overline{\text { Speed............................. } \quad \sim 100 \mathrm{~km} \mathrm{~s}^{-1} \quad \text { This paper }}$

a See $\S 4.2 .1$.

features, but it is spread over a much smaller velocity range. Hence, it is expected to be significantly more optically thick than the HV1 and HV2 components, making the mass determination more uncertain. Fitting this component as above, we can find reasonable fits with $T_{\mathrm{ex}} \sim 26-33 \mathrm{~K}, \tau_{32}$ about $7.5-10$, and a mass of about $0.035-0.042 M_{\odot}$. The corresponding optical depth of the $J=1-0$ line, $\tau_{10}$, is $\sim 2.2-2.6$ in these models; hence, any significant reduction in the total mass of the shell reduces $\tau_{10}$ below unity in large parts of the emitting region, resulting in a value of $R 13$ that is too low compared to the observed value of 0.12 . For example, if we reduce $\tau_{10}$ by a factor 2 , we get $R 13=0.08$, inconsistent with our data. Thus, the minimum mass of the lowvelocity component is about $0.035 M_{\odot}$.

Our data support the relatively high values of $T_{\mathrm{ex}}$ for the lowvelocity component derived from our modeling. The relatively high surface brightness observed at the systemic velocity (2.2 Jy beam $^{-1}$, Fig. 3) implies a brightness temperature of about $12 \mathrm{~K}$. Accounting for a beam-dilution factor of 2 (since the source size is about the beam size), the actual source brightness temperature is $T_{b}=24 \mathrm{~K}$, and the corresponding excitation temperature required for optically thick emission is $32 \mathrm{~K}$.

Thus, the total molecular mass is $M_{\mathrm{tot}}=0.065 M_{\odot}$. This is much lower than the value $\left(\sim 4.7 M_{\odot}\right)$ estimated from the dust mass $\left(\sim 0.023 M_{\odot}\right)$ inferred from a model of I22036's near- to farinfrared fluxes (as observed by Infrared Space Observatory [ISO], Midcourse Space Experiment [MSX], and IRAS; S03), assuming a gas-to-dust ratio $\delta=200$. In this model, cool (35-67 K) dust resides in a large spherical shell (with inner and outer radii of $1.4 \times 10^{17}$ and $\left.5.3 \times 10^{17} \mathrm{~cm}\right)$ of radial optical depth $A_{V} \sim 1.5$, and hot $(350-1000 \mathrm{~K})$ dust resides in a very optically thick $\left(A_{V} \sim 120\right)$, compact $\left[(3-4.6) \times 10^{14} \mathrm{~cm}\right]$ central torus. The mass of the cool shell is $4.7 M_{\odot}$, and the (very poorly constrained) mass of the compact central torus is $6 \times 10^{-4} M_{\odot}$, assuming a $100 \mu \mathrm{m}$ dust emissivity of $34 \mathrm{~cm}^{2} \mathrm{~g}^{-1}$. An additional warm $(82-145 \mathrm{~K})$ shell (with inner and outer radii of $1.3 \times 10^{16}$ and $5.7 \times 10^{16} \mathrm{~cm}$ ) with relatively little mass $\sim 0.005 M_{\odot}$ is added to the first two components to obtain a good fit to the spectral energy distribution (SED) in the $\sim 20-40 \mu \mathrm{m}$ range.

The size of the $\mathrm{CO} J=3-2$ emission source along its long axis is $\sim 5^{\prime \prime}$, which is significantly smaller than the central cavity (of size $\sim 9^{\prime \prime}$ ) in the model cool dust shell. Thus, the observed CO $J=3-2$ emission does not probe the material in the cool shell, which explains the discrepancy between the mass estimate based on the CO data $\left(\sim 0.032 M_{\odot}\right)$ and that derived from the dust emission $\left(4.7 M_{\odot}\right)$. The size of the dust shell is large (inner and outer radii of 4". 6 and 17".6) compared to the typical size of structures, which would not be largely resolved out by the interferometer, which is $\sim 9^{\prime \prime}$ given our minimum UV spacing of $10 \mathrm{k} \lambda$, and good UV coverage for spacings greater or equal to about $23 \mathrm{k} \lambda$. Hence, it is likely that a significant fraction of the $\mathrm{CO} J=3-2$ flux from this shell is resolved out by our interferometric observations. Furthermore, astrophysical mechanisms for the lack of observable CO $J=3-2$ emission from the dust shell are also plausible, e.g., (1) photodissociation of $\mathrm{CO}$ due to the interstellar ultraviolet radiation, and (2) insufficient excitation due to low gas kinetic temperatures. We think it is unlikely that photodissociation is important, since the photodissociation radius (estimated using Table 3 from Mamon et al. 1988) given the high mass-loss rate inferred for the shell $\left(5.5 \times 10^{-4} M_{\odot} \mathrm{yr}^{-1}\right.$; S03 $)$, and an assumed expansion velocity of $15 \mathrm{~km} \mathrm{~s}^{-1}$, is a few $\times 10^{18} \mathrm{~cm}$, which is significantly larger than the outer radius of the dust shell. As in the case of other PPNs (e.g., IRAS 19024+0044, Sahai et al. 2005b; Roberts 22 [MR 22], Sahai et al. 1999), it is plausible that the gas kinetic temperatures in the extended dust shell of I22036 are quite low because of the combined effect of cooling due to adiabatic expansion and the lack of dust frictional heating.

\subsection{The Central Submillimeter Continuum Source: Large Grains in I22036's Dusty Torus}

The 0.88 and $2.6 \mathrm{~mm}$ fluxes reported here are significantly in excess of the SED resulting from the dust model of S03. This can be seen in Figure 8, which shows a modified version of the S03 model (discussed below); the SMA and OVRO data points lie significantly above the dashed red curve representing emission 


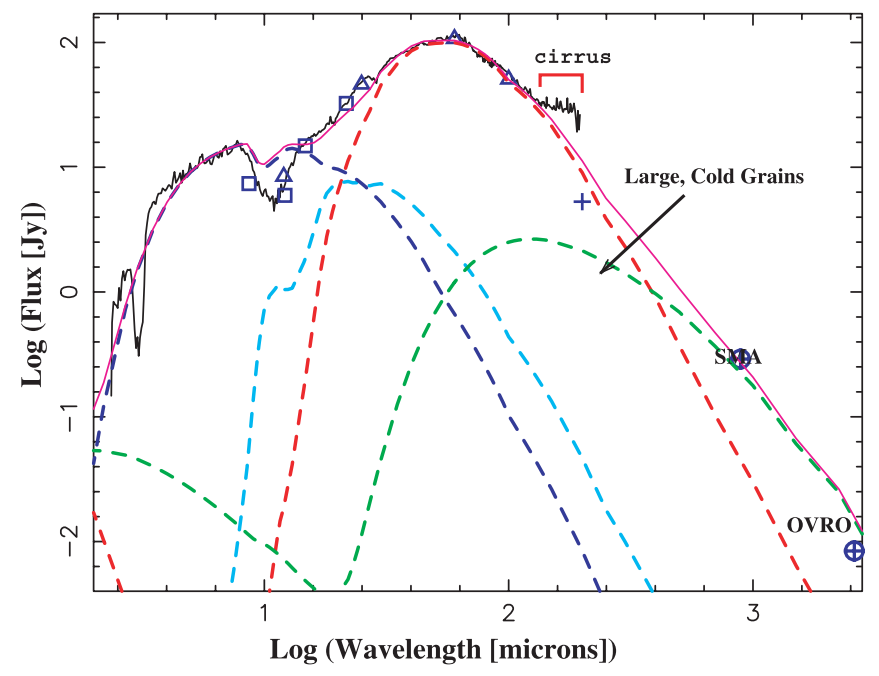

FIG. 8.-Observations (ISO spectra [black curve] and blue photometric data: SMA $0.88 \mathrm{~mm}$ and OVRO $2.6 \mathrm{~mm}$ [blue circles with plus signs], MSX [blue squares], IRAS [blue triangles], and ISO PHOT: [blue plus sign]) and a model spectrum (magenta curve) of I22036. Individual components (dashed curves) of the model are also shown: cool (red curve) and warm (cyan curve) shells, hot inner disk (blue curve), and a component with large, cold grains (green curve), probably associated with I22036's dusty waist. The flattened region at the long-wavelength end of the ISO spectrum is most likely due to contamination from interstellar cirrus emission.

from the cool shell, which is the dominant component at long wavelengths in the S03 model. Since the observed $0.88 \mathrm{~mm}$ continuum source is unresolved, its size is less than $0.9 \times 0.7$ (the FWHM of the SMA clean beam). Thus, the $0.88 \mathrm{~mm}$ flux in the S03 model, within this small aperture, would be even lower than shown by the dashed red curve in Figure 8. Since the spectral index of the continuum from 0.88 to $2.6 \mathrm{~mm}, \alpha$ (defined as $F_{\nu} \sim \nu^{\alpha}$ ), is 3.3, too steep to be a result of thermal brehmstrahhlung, the submillimeter- and millimeter-wave fluxes in I22036 are most likely the result of thermal emission from a population of quite large, cold grains. We believe that the grains must be large because the opacity spectral index $\beta=d \ln \kappa / d \ln \nu$ in the submillimeter and millimeter-wavelength region from our data ${ }^{7}$ is 1.3 , which is significantly smaller than the value expected for standard small grains $(\beta \approx 2)$. Such small values of $\beta$ are consistent with those obtained in theoretical models with grain sizes (in a power-law size distribution) extending to the millimeter range (Draine 2006). Given the compact nature of the $0.88 \mathrm{~mm}$ continuum source, it is quite likely that these large grains are physically located within the dusty waist seen in the HST images. Note that the bulk of the grains producing the SED of I22036 in the $\sim$ few to $200 \mu \mathrm{m}$ range is probably small $(\sim 0.1 \mu \mathrm{m})$, as has been found for other PPNs from the very large fractional polarizations typically observed toward these objects at optical and near-IR wavelengths (e.g., Sahai et al. 1998a, 1999; Oppenheimer et al. 2005).

In order to provide rough constraints on the physical parameters of the large-grain dust source, we have used DUSTY (as in S03) to compute the emission from a dust shell with large grains, varying its major inputs - the temperature at the shell inner radius (the ratio of the outer to inner radius is arbitrarily assumed to be 2), the optical depth at $100 \mu \mathrm{m}, \tau(100)$, the grain temperature at the inner radius $T_{d}($ in), and the grain size, $a$ - to fit the observed SMA and OVRO continuum fluxes. We find that dust

\footnotetext{
${ }^{7}$ Derived assuming optically thin emission in the Rayleigh-Jeans limit, for which $\alpha=2+\beta$.
}

grains with $a \gtrsim 1 \mathrm{~mm}$ and $T_{d}$ (in) $\lesssim 50 \mathrm{~K}$ are needed for producing the observed fluxes at 0.88 and $2.6 \mathrm{~mm}$. The shell has an optical depth $\tau(100)=0.1$. Models with smaller grains (e.g., $a \lesssim 500 \mu \mathrm{m}$ ), which fit the 0.88 and $2.6 \mathrm{~mm}$ fluxes, produce too much flux in the $\sim 100-200 \mu \mathrm{m}$ range. In order for the large-grain shell to be smaller than or equal to the observed size of the $0.88 \mathrm{~mm}$ continuum source, i.e., $<2.5 \times 10^{16} \mathrm{~cm}$, the source luminosity corresponding to the required irradiating intensity must be $\$ 350 L_{\odot}$, i.e., $\sim 15 \%$ of the total source luminosity. This result requires that the large grains be confined to a region where they can be substantially shielded from the full radiation of the central star, supporting our conjecture that they are located within the toroidal waist seen in the HST images of I22036, since this region is partially shielded from the full stellar radiation field by the compact, optically thick central dust component inferred by S03. From our model, we estimate the mass in dust of this large grain component to be $\sim 0.04 M_{\odot}$, taking the dust mass extinction coefficient, $\kappa$, at $\lambda \ll a$ (say at $100 \mu \mathrm{m}$ ) to be $2.5 \mathrm{~cm}^{2} \mathrm{~g}^{-1}$, derived from $\kappa=\sigma\left(4 \pi a^{3} \rho_{d} / 3\right)^{-1}$, with $\sigma=\pi a^{2}, a=1 \mathrm{~mm}$, and $\rho_{d}=3 \mathrm{~g} \mathrm{~cm}^{-3}$.

Our dust model is based on commonly used values for the wavelength-dependent complex refractive index of silicate dust particles; however, uncertainties in the dust mass absorption coefficient may allow for a reduction of the mass estimate by as much as a factor of 2 . Thus, it is clear that there is a very substantial mass of such grains in the dusty waist of I22036. If we assume a gas-to-dust ratio of 200 in the waist, as in the cool dust shell, the total mass in the toroid becomes 4-8 $M_{\odot}$, a disturbingly large value, especially when combined with the $4.7 M_{\odot}$ of material in the cool dust shell. We therefore conclude that the gas content in the waist must be significantly depleted, and the gasto-dust ratio consequently much lower than the typical value, maybe by as much as an order of magnitude.

\section{THE ${ }^{13} \mathrm{C}$ TO ${ }^{12} \mathrm{C}$ ISOTOPE RATIO}

We derive the ${ }^{13} \mathrm{C} /{ }^{12} \mathrm{C}$ abundance ratio, $f_{13 / 12}$, in 22036 from the ratio of the $J=1-0{ }^{13} \mathrm{CO}$ and ${ }^{12} \mathrm{CO}$ fluxes. The difference in the shapes of the ${ }^{12} \mathrm{CO}$ and ${ }^{13} \mathrm{CO}$ lines shown in Figure 1 is consistent with a uniform value of $f_{13 / 12}$, but with larger optical depths in the central component compared to the high-velocity component. As indicated by the prominent core emission seen in the ${ }^{13} \mathrm{CO}$ but not the ${ }^{12} \mathrm{CO}$ line profile, the ratio of the ${ }^{13} \mathrm{CO}$ to ${ }^{12} \mathrm{CO}$ flux in the core ( 0.37 ) is much higher than that in the high-velocity wings $(0.2)$. This difference in the flux ratio between the low- and high-velocity outflows is due to the difference in their optical depths: $\tau_{10}$ is about 2 in the central component, but 0.4 in the HV1 component (see $\S 5$ ). We have therefore computed $f_{13 / 12}$ from the flux ratio in the more optically thin part of the lines; the ${ }^{13} \mathrm{CO} /{ }^{12} \mathrm{CO}$ flux ratio of 0.2 gives $f_{13 / 12}=0.16$. We find that the same value of $f_{13 / 12}$ reproduces the observed ${ }^{13} \mathrm{CO} /{ }^{12} \mathrm{CO}$ flux ratio in the core if $\tau_{10}=$ 2.2 and $T_{\text {ex }}=30 \mathrm{~K}$, supporting our modeling of the ${ }^{12} \mathrm{CO}$ emission from the low-velocity outflow.

Thus, the ${ }^{13} \mathrm{C} /{ }^{12} \mathrm{C}$ abundance ratio in $\mathrm{I} 22036$ is close to the maximum value achieved in equilibrium $\mathrm{CNO}$ nucleosynthesis (0.33), and provides a strong test of models of stellar nucleosynthesis. For comparison, other evolved objects that show high values of $f_{13 / 12}$ (i.e., in excess of 0.1 ) are (1) the circumstellar envelopes of some oxygen-rich AGB or post-AGB objects, such as $\mathrm{OH} 231.8+4.2$ (Sánchez Contreras et al. 2000), Frosty Leo (Sahai et al. 2000), and U Equ (Geballe et al. 2005), (2) the rare class of $J$-type carbon stars, e.g., Y CVn or T Lyr (Lambert et al. 1986), and (3) selected PNs, e.g., M1-M16 (Sahai et al. 1994). In general, there is increasing observational evidence that a large 
fraction of evolved stars have ${ }^{12} \mathrm{C} /{ }^{13} \mathrm{C}$ abundance ratios lower than about 15 , significantly lower than those predicted by standard models. For example, from millimeter-wave CO studies, Palla et al. (2000) and Balser et al. (2002) have estimated the $f_{13 / 12}$ ratio in modest PN samples (16 and 9 objects, respectively) and find it lies typically in the range $\sim 0.04-0.1$.

There are two processes that can enhance the $f_{13 / 12}$ ratio above the values predicted by the standard model. The first, cold-bottomprocessing (CBP), is a deep mixing mechanism believed to operate during the red giant branch (RGB) phase of stars with mass less than about $2 M_{\odot}$ (Wasserburg et al. 1995), and the degree of enhancement decreases with increasing stellar mass. In a $1 M_{\odot}$ star, CBP can produce a value of $f_{13 / 12}$ as high as 0.2 during the RGB phase, but this ratio is lowered to $0.025-0.05$ during the AGB phase due to the addition of fresh ${ }^{12} \mathrm{C}$. Since the progenitor central star of I22036 is significantly more massive than $2 M_{\odot}$ considering the high mass of its dust shell, CBP cannot account for its high $f_{13 / 12}$ ratio.

The second mechanism, hot-bottom-burning (HBB), is believed to operate in AGB stars with initial masses above about 3.5-4 $M_{\odot}$. Such stars develop deep convective envelopes with very high temperatures at the base, causing the operation of the $\mathrm{CN}$ cycle (i.e., ${ }^{12} \mathrm{C} \rightarrow{ }^{13} \mathrm{C} \rightarrow{ }^{14} \mathrm{~N}$ ). In this case, $f_{13 / 12}$ will be near the equilibrium ratio of 0.33 , and the surface $f_{13 / 12}$ is expected to asymptotically approach this limit. HBB is thus the likely mechanism for producing the high $f_{13 / 12}$ ratio in I22036. Rubin et al. (2004) reach a similar conclusion for the PN 2440, where they find $f_{13 / 12}$ $\sim 0.2$ from analysis of $\mathrm{C}$ III lines in International Ultraviolet Explorer (IUE) data.

\section{DISCUSSION}

\subsection{The Shaping of Bipolar Planetary Nebulae}

The dynamical evolution and shaping of PPNs is believed to result from the shock interaction between a fast, collimated postAGB wind and the slowly expanding, dense wind ejected during the previous AGB phase. The bipolar post-AGB wind impinges on, and thus accelerates and heats, two localized regions of the (presumably spherical) AGB wind. A significant amount of momentum is believed to be transferred to the rest of the AGB shell by means of bowlike shocks. Although morphological identifications of bow shocks have been made in PPNs, their characteristic spatiokinematic structure has seldom been identified. Thus, the bow-shock structures inferred kinematically from our CO $J=3-2$ imaging of I22036 provide support for, and are an important input for quantitative modeling of the physics of, the interaction of collimated fast winds with AGB envelopes as a production mechanism for bipolar PPNs (see, e.g., LS03).

We have shown that the bipolar molecular outflow in I22036 cannot be driven by radiation pressure. Bujarrabal et al. (2001) infer the presence of fast molecular outflows in a large sample of PPNs, from the extended wings seen in their $\mathrm{CO}$ line profiles, and conclude that these outflows cannot be driven by radiation pressure. Only for a small fraction of objects in their sample (e.g., CRL 2688 or the Egg Nebula, Cox et al. 2000; IRAS 09371+ 1212 or the Frosty Leo Nebula, Castro-Carrizo et al. 2005; CRL 618, Sánchez Contreras et al. 2004; OH 231.8+4.2, Alcolea et al. 2001) has interferometric mapping been carried out, directly revealing the structure of the fast outflow. In all such well-mapped PPNs, the fast outflow or each of its components (if it comprises multiple components) appears to be collimated and directed along well-defined axes. Our results show that I22036 fits well in this picture of the fast molecular outflows in PPNs. Given the in- creasing direct evidence for the presence of jetlike outflows in very young PPNs (e.g., W43A, Imai et al. 2002; IRAS 16342-3814, Sahai et al. 2005a; IRAS 19134+2131, Imai et al. 2004), we believe that the case for the fast molecular outflows in I22036 and other PPNs being driven by collimated jets is quite strong. The presence of such jets, operating in an episodic fashion and changing their directionality, has been proposed as a general mechanism for explaining the diverse morphologies and widespread presence of point symmetry in PNs (Sahai \& Trauger 1998; Sahai 2004). The launching mechanism for such jets remains unknown, but given the similarity between the fast outflow speeds in PPNs and jets in low-mass pre-main-sequence stars with accretion disks, it is plausible that the mechanisms that drive jets in PPNs and young stellar objects (YSOs) are very similar. In this scenario, the accretion disk in a PPN would be produced around a relatively close companion low-mass star, which accretes material from the dense AGB wind of the primary (Morris 1987; Mastrodemos \& Morris 1998).

Our CO $J=3-2$ observations and the dust model of S03 indicate an apparent absence of dense cool material in I22036 along nonpolar directions, in the volume between the molecular outflow, and the inner radius of the cool dust shell (hereafter, the "in-between" region). In the S03 model, the inferred inner radius of this dust shell cannot be significantly smaller than the derived value because I22036's SED (which peaks near $60 \mu \mathrm{m}$ ) dictates that the emitting grains have low temperatures and therefore be located relatively far from the illuminating source. Even if we explicitly account for the reduction in the illuminating flux due to the dusty torus in S03's model, which covers $\sim 0.4$ of the $4 \pi$ solid angle around the central star, the lower limit to the fraction of the central star's total luminosity, which irradiates the cool dust shell, is 0.6. Hence, the inner radius of the shell (which scales as the square root of the illuminating flux) is at most $23 \%$ smaller than that derived by S03. Although one may explain the absence of $\mathrm{CO}$ emission using insufficient excitation as an argument, the lack of thermal dust emission from the inbetween region is strong evidence for the lack of dense material there.

A faint halo has been found in $\mathrm{I} 22036$ at $0.8 \mu \mathrm{m}$ in new deep images taken with the the HST Advanced Camera for Surveys (ACS; Sahai et al. 2007, in preparation), which indicates the presence of a rather tenuous surrounding remnant AGB envelope. But we do not know if the fast molecular outflow in I22036 was produced by a collimated jet interacting with this tenuous envelope. If the ambient material is of sufficiently low density, then the swept-up shell may be too faint to be observable, and one could then see the jet material directly, as appears to be the case for the young PN, Hen 2-90 (Sahai \& Nyman 2000; Lee \& Sahai 2004). Only detailed numerical simulations of the jet-AGB envelope interaction in I22036 can tell us if the material in its fast molecular outflow (1) represents the swept-up shell due to the interaction of the jet with the tenuous AGB envelope seen in the near-IR halo, (2) is intrinsic, i.e., belongs to the underlying jet itself, or (3) represents material associated with the dense torus that has been accelerated by the jet. In model 3 , one can imagine that the jet punched its way out of a flattened dusty cocoon around the central star, as envisaged for the case of the well-studied PPN, CRL 2688 (Sahai et al. 1998b), where cocoon matter entrained by the jet produces the observed bipolar outflow in $\mathrm{CO}$ emission, and the remnant cocoon represents the torus.

While considering the above issues, it is useful to note that the nebular properties of I22036 are very similar to that of the wellstudied object $\mathrm{OH} 231.8+4.2$, although their central stars have 
different spectral types ( F5 vs. M9, respectively). As in I22036, $\mathrm{OH} 231.8$ harbors a massive molecular bipolar outflow oriented along the long axis of the optical bipolar nebula, and a weak round near-IR halo is seen around its bipolar outflow, signifying the presence of a tenuous spherical envelope surrounding this outflow (i.e., characterized by a mass-loss rate of $\sim 10^{-6} M_{\odot} \mathrm{yr}^{-1}$; Alcolea et al. 2001). Furthermore, I22036 also has a very high ${ }^{13} \mathrm{C} /{ }^{12} \mathrm{C}$ ratio, similar to that in $\mathrm{OH} 231$.8. Thus, it appears that both $\mathrm{I} 22036$ and $\mathrm{OH} 231.8$ are very similar objects and are formed by a similar mechanism; the main difference between the two is that $\mathrm{OH} 231.8+$ 4.2 is a more extreme version - in it, the mass of cool material in the bipolar outflow is estimated to be significantly larger (Alcolea et al. 2001). Both objects show high-velocity OH maser emission. Since $\mathrm{OH} 231.8$ was not observed by $I S O$, we do not know its far-IR SED as well as that of I22036; however, the existing IRAS data show that the SEDs of both objects are very similar. For example, in OH 231.8 (as in I22036) (1) the broadband IRAS fluxes from 12 to $100 \mu \mathrm{m}$ show a peak at $60 \mu \mathrm{m}$, and (2) the LowResolution Spectrograph (LRS) data show a broad silicate absorption feature at $10 \mu \mathrm{m}$ and a steeply rising spectrum toward longer wavelengths. OH 231.8 also shows a compact continuum component at $1.3 \mathrm{~mm}$ (Sánchez Contreras et al. 1998), which the authors interpret as emission from grains larger than about $5 \mu \mathrm{m}$.

I22036 most likely had a fairly massive progenitor, easily $\gtrsim 4 M_{\odot}$. The combination of the high circumstellar mass (i.e., in the extended dust shell and the torus) and the high ${ }^{13} \mathrm{C} /{ }^{12} \mathrm{C}$ ratio in this object provides strong support for the operation of the HBB mechanism.

\subsection{Dusty Waists and Large Grains}

Compared to the question of forming bipolar and multipolar morphologies in PPNs, the formation of the dense, dusty waists in these objects remains a much bigger puzzle. The presence of large grains with a very substantial mass associated with I22036's toroidal waist is a surprising but not unprecedented result. In the well-studied PPN, AFGL 2688, Jura et al. (2000) find evidence for a substantial mass $\left(\sim 0.01 M_{\odot}\right)$ of large-sized grains. In the Red Rectangle (RR; the nearest PPN to us at a distance of $350-700 \mathrm{pc}$, bipolar in morphology, and harboring a disk in rotation around a central binary), Jura et al. (1997) derive a minimum mass of $5 \times$ $10^{-4} M_{\odot}$ in large $(a \gtrsim 0.02 \mathrm{~cm}), \operatorname{cool}(50 \mathrm{~K})$ grains based on RR's $1.3 \mathrm{~mm}$ continuum flux. Men'shchikov et al. (2002) fit the full SED and near-IR images of the RR using a $2 \mathrm{D}$ dust radiative transfer model and infer a dust mass of about $0.01 M_{\odot}$ in grains with $a \sim 1 \mathrm{~mm}$ in a compact, central torus. In the quadrupolar PPN IRAS 19475+3119, Sarkar \& Sahai (2006) find from a comparison of a detailed model of its full SED (from optical wavelengths to $200 \mu \mathrm{m}$ ) to the observed $0.85 \mathrm{~mm}$ flux that there is a substantial $0.85 \mathrm{~mm}$ excess flux, which also requires the presence of large, cool grains. We speculate that the formation of dusty waists and the presence of large grains in PPNs are due to intimately linked physical processes, and thus a resolution of the issue of waist formation may well lead to a resolution of how the large grains are made, or vice versa. One possible mechanism is the destruction of volatile cometary-debris disks within several hundred to a thousand AU (Stern et al. 1990) by an intermediate-mass star during its luminous post-main-sequence evolution, providing a source of large solid particles that could form a dusty torus. However, the total mass of large grains that we have found in I22036 is far too large compared to the mass estimates for our Kuiper Belt
( $\sim 0.1 M_{\oplus}$; e.g., Luu \& Jewitt 2002) or the inner parts of the Oort Cloud ( $\sim 40 M_{\oplus}$; e.g., Dones et al. 2004), and argues against such a process, unless of course the Kuiper Belt/Oort Cloud analogs of an intermediate-mass star, such as that in I22036, are significantly more massive than those in our solar system.

\section{CONCLUSIONS}

Using the SMA, we have made high angular resolution $\left(\sim 1^{\prime \prime}\right)$ maps of the CO $J=3-2$ emission in IRAS $22036+5306$, a bipolar preplanetary nebula (PPN) with knotty jets, discovered previously in one of our HST imaging surveys of candidate PPNs. These data have been supplemented by lower-resolution $\mathrm{CO}$ and ${ }^{13} \mathrm{CO} J=1-0$ observations with OVRO, as well as optical longslit echelle spectroscopy. We find

1. The $\mathrm{CO} J=3-2$ spatially integrated line profile covers a total velocity extent (FWZI) of $\sim 500 \mathrm{~km} \mathrm{~s}^{-1}$. The $\mathrm{CO}$ and ${ }^{13} \mathrm{CO}$ $J=1-0$ also show large velocity widths, although with a smaller spread due to the lower sensitivity of these data.

2 . The $\operatorname{CO} J=3-2$ emission comes from a massive highvelocity bipolar outflow aligned with the optical nebulosity. The characteristic position-velocity (PV) signature of a bow shock resulting from a high-velocity bipolar collimated outflow/jet interacting with dense ambient material can be seen in the $\mathrm{CO}$ $J=3-2$ data in both the red- and blueshifted components of the outflow. The PV structure has a high degree of symmetry around the center, indicating that the bipolar outflow and surrounding medium are correspondingly symmetric. The bow shocks do not lie at the tips of the optical lobes.

3. The $\mathrm{H} \alpha$ line shows a profile with a central peak, very wide wings, and an absorption dip in the blue wing of the line, blueshifted from the systemic velocity by about $100 \mathrm{~km} \mathrm{~s}^{-1}$. The absorption feature indicates the presence of a fast, neutral outflow inside the lobes.

4. A central, unresolved, continuum source with a flux of $0.29 \pm 0.04 \mathrm{Jy}(8.4 \pm 1.7 \mathrm{mJy})$ at $\lambda=0.88 \mathrm{~mm}(2.6 \mathrm{~mm})$ is seen in the SMA and OVRO data.

5. The mass in the high-velocity outflow, i.e., at velocities offset more than $\pm 15 \mathrm{~km} \mathrm{~s}^{-1}$ from the systemic velocity, is $0.03 M_{\odot}$, and the scalar momentum is $1.1 \times 10^{39} \mathrm{~g} \mathrm{~cm} \mathrm{~s}^{-1}$. The large value of the scalar momentum, together with the small age of the highvelocity outflow, implies that it cannot be driven by radiation pressure.

6. The total molecular mass derived from integrating the flux of the CO $J=3-2$ line over its full velocity range is $\sim 0.065 M_{\odot}$, much lower than the value $\left(\sim 4.7 M_{\odot}\right)$ estimated previously from a dust-shell model of I22036's near- to far-IR fluxes, assuming a gas-to-dust ratio $\delta=200$. This is probably due to the interferometric $J=3-2$ observations resolving the flux from the large shell (inner and outer radii of $1.4 \times 10^{17}$ and $5.3 \times 10^{17} \mathrm{~cm}$ ) in which the cool dust resides. Furthermore, the gas in this shell may not be sufficiently excited.

7. The small size of the continuum source indicates that it is associated with the dusty toroidal waist of I22036. The submillimeter- and millimeter-wave continuum fluxes imply the presence of a very substantial mass $\left(\sim 0.02-0.04 M_{\odot}\right)$ of large (radius $\gtrsim 1 \mathrm{~mm}$ ) cold grains in this waist.

8. The ${ }^{13} \mathrm{C} /{ }^{12} \mathrm{C}$ ratio in $\mathrm{I} 22036$ is 0.16 , close to the maximum value achieved in equilibrium $\mathrm{CNO}$ nucleosynthesis $(0.33)$. The enhancement is most likely due to the operation of hot-bottomburning, which operates in stars with initial masses larger than about $4 M_{\odot}$. 
We thank Nick Sterling for pointing out the importance of hotbottom-burning in enhancing the ${ }^{13} \mathrm{C} /{ }^{12} \mathrm{C}$ ratio and Noam Soker for reading an earlier version of this paper. R. S. and M. M. thank NASA for partial financial support through an LTSA award (399.20.40.06). R. S. also thanks NASA for support from an
ADP award (399.20.00.08), as well as from an STScI HST award (GO 09463.01). C. S. C.'s work was supported by the National Science Foundation through grant 9981546 to Owens Valley Radio Observatory and the Spanish MCyT under project DGES/ AYA2003-2785 and the 2005 "Ramón y Cajal" program.
Alcolea, J., Bujarrabal, V., Sánchez Contreras, C., Neri, R., \& Zweigle, J. 2001, A\&A, 373, 932

Balser, D. S., McMullin, J. P., \& Wilson, T. L. 2002, ApJ, 572, 326

Bujarrabal, V., Castro-Carrizo, A., Alcolea, J., \& Sánchez Contreras, C. 2001, A\&A, 377, 868

Castro-Carrizo, A., Bujarrabal, V., Sánchez Contreras, C., Sahai, R., \& Alcolea, J. 2005, A\&A, 431, 979

Cox, P., Lucas, R., Huggins, P. J., Forveille, T., Bachiller, R., Guilloteau, S., Maillard, J. P., \& Omont, A. 2000, A\&A, 353, L25

Dones, L., Weissman, P. R., Levison, H. F., \& Duncan, M. J. 2004, in ASP Conf. Ser. 323, Star Formation in the Interstellar Medium, ed. D. Johnstone et al. (San Francisco: ASP), 371

Draine, B. T. 2006, ApJ, 636, 1114

Geballe, T. R., Barnbaum, C., Noll, K. S., \& Morris, M. 2005, ApJ, 624, 983

Imai, H., Morris, M., Sahai, R., Hachisuka, K., \& Azzollini F., J. R. 2004 A\&A, 420, 265

Imai, H., Obara, K., Diamond, P. J., Omodaka, T., \& Sasao, T. 2002, Nature, 417,829

Jura, M., Turner, J. L., \& Balm, S. P. 1997, ApJ, 474, 741

Jura, M., Turner, J. L., Van Dyk, S., \& Knapp, G. R. 2000, ApJ, 528, L105

Lambert, D. L., Gustafsson, B., Eriksson, K., \& Hinkle, K. H. 1986, ApJS, 62, 373

Lee, C-F., \& Sahai, R. 2003, ApJ, 586, 319 (LS03)

2004, ApJ, 606, 483

Luu, J. X., \& Jewitt, D. C. 2002, ARA\&A, 40, 63

Mamon, G. A., Glassgold, A. E., \& Huggins, P. J. 1988, ApJ, 328, 797

Mastrodemos, N., \& Morris, M. 1998, ApJ, 497, 303

Men'shchikov, A. B., Schertl, D., Tuthill, P. G., Weigelt, G., \& Yungelson, L. R. 2002, A\&A, 393, 867

Morris, M. 1987, PASP, 99, 1115

Oppenheimer, B. D., Bieging, J. H., Schmidt, G. D., Gordon, K. D., Misselt, K. A., \& Smith, P. S. 2005, ApJ, 624, 957

Palla, F., Bachiller, R., Stanghellini, L., Tosi, M., \& Galli, D. 2000, A\&A, 355, 69

\section{EFERENCES}

Rubin, R. H., Ferland, G. J., Chollet, E. E., \& Horstmeyer, R. 2004, ApJ, 605, 784 Sahai, R. 2004, in ASP Conf. Ser. 313, Asymmetrical Planetary Nebulae III, ed. M. Meixner et al. (San Francisco: ASP), 141

Sahai, R., Bujarrabal, V., Castro-Carrizo, A., \& Zijlstra, A. 2000, A\&A, 360, L9 Sahai, R., Hines, D. C., Kastner, J. H., Weintraub, D. A., Trauger, J. T., Rieke,

M. J., Thompson, R. I., \& Schneider, G. 1998a, ApJ, 492, L163

Sahai, R., Le Mignant, D., Sánchez Contreras, C., Campbell, R. D., \& Chaffee, F. H. 2005a, ApJ, 622, L53

Sahai, R., \& Nyman, L.-Å. 2000, ApJ, 538, L145

Sahai, R., \& Sánchez Contreras, C. 2004, in ASP Conf. Ser. 313, Asymmetrical Planetary Nebulae III, ed. M. Meixner et. al. (San Francisco: ASP), 32

Sahai, R., Sánchez Contreras, C., \& Morris, M. 2005b, ApJ, 620, 948

Sahai, R., \& Trauger, J. T. 1998, AJ, 116, 1357

Sahai, R., Wootten, A., Schwarz, H. E., \& Wild, W. 1994, ApJ, 428, 237

Sahai, R., Zijlstra, A., Bujarrabal, V., \& te Lintel Hekkert, P. 1999, AJ, 117, 1408

Sahai, R., Zijlstra, A., Sánchez Contreras, C., \& Morris, M. 2003, ApJ, 586, L81 (SCZM03)

Sahai, R., et al. 1998b, ApJ, 493, 301

Sánchez Contreras, C., Alcolea, J., Bujarrabal, V., \& Neri, R. 1998, A\&A, 337, 233

Sánchez Contreras, C., Bujarrabal, V., Castro-Carrizo, A., Alcolea, J., \& Sargent, A. 2004, ApJ, 617, 1142

Sánchez Contreras, C., Bujarrabal, V., Neri, R., \& Alcolea, J. 2000, A\&A, 357, 651 Sánchez Contreras, C., \& Sahai, R. 2001, ApJ, 553, L173

. 2004, in ASP Conf. Ser. 313, Asymmetrical Planetary Nebulae III, ed. M. Meixner et al. (San Francisco: ASP), 377

Sarkar, G., \& Sahai, R. 2006, ApJ, 644, 1171

Stern, S. A., Shull, J. M., \& Brandt, J. C. 1990, Nature, 345, 305

van de Steene, G. C., Wood, P. R., \& van Hoof, P. A. M. 2000, in ASP Conf. Ser. 199, Asymmetrical Planetary Nebulae II: From Origins to Microstructures, ed. J. H. Kastner, N. Soker, \& S. Rappaport (San Francisco: ASP), 191

Wasserburg, G. J., Boothroyd, A. I., \& Sackmann, I.-J. 1995, ApJ, 447, L37 Zijlstra, A. A., Chapman, J. M., te Lintel Hekkert, P., Likkel, L., Comeron, F., Norris, R. P., Molster, F. J., \& Cohen, R. J. 2001, MNRAS, 322, 280 